\title{
Naringenin improves learning and memory in an Alzheimer's disease rat model: Insights into the underlying mechanisms
}

\author{
Saeed Ghofrani, Mohammad-Taghi Joghataei, Simin Mohseni, Tourandokht \\ Baluchnejadmojarad, Maryam Bagheri, Safoura Khamse and Mehrdad Roghani
}

\section{Linköping University Post Print}

\section{Tweet}

N.B.: When citing this work, cite the original article.

Original Publication:

Saeed Ghofrani, Mohammad-Taghi Joghataei, Simin Mohseni, Tourandokht Baluchnejadmojarad, Maryam Bagheri, Safoura Khamse and Mehrdad Roghani, Naringenin improves learning and memory in an Alzheimer's disease rat model: Insights into the underlying mechanisms, 2015, European Journal of Pharmacology, (764), 195-201.

http://dx.doi.org/10.1016/j.ejphar.2015.07.001

Copyright: Elsevier

http://www.elsevier.com/

Postprint available at: Linköping University Electronic Press

http://urn.kb.se/resolve?urn=urn:nbn:se:liu:diva-120716 


\section{Naringenin improves learning and memory in an Alzheimer's disease rat}

\section{model: insights into the underlying mechanisms}

Saeed Ghofrani a,b ${ }^{\text {, }}$ Mohammad-Taghi Joghataei ${ }^{\text {b }}$, Simin Mohseni ${ }^{\text {a }}$, Tourandokht

Baluchnejadmojarad ${ }^{\text {d, }}$, Maryam Bagheri ${ }^{\text {a,c }}$, Safoura Khamse ${ }^{\text {e }}$, Mehrdad Roghani ${ }^{\text {f,* }}$

${ }^{\text {a }}$ Department of Clinical and Experimental Medicine, Faculty of Health Sciences, Linköping

University, Linköping, Sweden

${ }^{\mathrm{b}}$ Division of Neuroscience, Cellular and Molecular Research Center, Iran University of Medical Sciences, Tehran, Iran.

${ }^{c}$ Department of Physiology, School of Medicine, Ilam University of Medical Sciences, Ilam, Iran.

${ }^{\mathrm{d}}$ Department of Physiology, School of Medicine, Iran University of Medical Sciences, Tehran, Iran.

${ }^{\mathrm{e}}$ Department of Physiology, School of Medicine, Tehran University of Medical Sciences, Tehran, Iran.

${ }^{\mathrm{f}}$ Neurophysiology Research Center, Shahed University, Tehran, Iran.

* To whom correspondence should be addressed:

Dr. Mehrdad Roghani

Neurophysiology Research Center, Shahed University, Tehran, Iran.

Fax: +98 21 88966310; E-mail: mroghani@shahed.ac.ir 


\begin{abstract}
Alzheimer's disease (AD) is one of the prevalent neurological disorders of the central nervous system hallmarked by increased beta-amyloid $(\mathrm{A} \beta)$ deposition and ensuing learning and memory deficit. In the present study, the beneficial effect of naringenin on improvement of learning and memory was evaluated in an Alzheimer's disease rat model. The A $\beta$-injected rats showed a lower alternation score in Y-maze task, impairment of retention and recall capability in passive avoidance test, and lower correct choices and higher errors in radial arm maze (RAM) task as compared to sham group in addition to enhanced oxidative stress and apoptosis. Naringenin, but not a combination of naringenin and fulverstrant (an estrogenic receptor antagonist) significantly improved the performance of $\mathrm{A} \beta$-injected rats in passive avoidance and RAM tasks. Naringenin pretreatment of $\mathrm{A} \beta$-injected rats also lowered hippocampal malondialdehyde (MDA) with no significant effect on nitrite and superoxide dismutase (SOD) activity in addition to lowering apoptosis. These results suggest naringenin pretreatment attenuates $A \beta$-induced impairment of learning and memory through mitigation of lipid peroxidation and apoptosis and its beneficial effect is somewhat mediated via estrogenic pathway.
\end{abstract}

Keywords: Naringenin, Alzheimer's disease, Beta-Amyloid, Learning and Memory, Oxidative stress, Apoptosis 


\section{Introduction}

Alzheimer's disease (AD) is known as the most causative factor for dementia and well characterized by the aggregated $\beta$-amyloid (A $\beta$ ) (Bao et al., 2013). AD is a progressive neurodegenerative disorder that with time impairs cognitive skills and learning and memory abilities (Mimura, 2008). The main pathogenic mechanisms responsible for AD include cholinergic dysfunction, enhanced oxidative stress burden and disturbed antioxidant defense system, augmented inflammatory response, and an excitotoxic insult (Grothe et al., 2014;

Obulesu and Jhansilakshmi, 2014; Subash et al., 2014). Currently, there is no effective cure for $\mathrm{AD}$, so the focus of treatments is on stopping or slowing the progressive decline in cognitive functions (Zhu et al., 2013).

Naringenin is a natural flavanone, richly found in citrus and grape fruits, exhibits antioxidant potential, improves brain insulin signaling and cognitive functions and ameliorates AD-type neurodegeneration due to intracerebroventricular-streptozotocin (Khan et al., 2012; Yang et al., 2014). In addition, naringenin exhibits anti-inflammatory effect (Esmaeili and Alilou, 2014), exerts neuroprotective effect in 6-hydroxydopamine (6-OHDA)-induced model of Parkinson's disease and also against 6-OHDA neurotoxicity (Lou et al., 2014; Zbarsky et al., 2005). Of interest, cholinergic function is improved by naringenin due to its antioxidant property and through inhibition of cholinesterase activity in the hippocampal region, in this way could improve type-2 diabetes-induced memory dysfunction (Rahigude et al., 2012). Based on these findings, we tried to evaluate the protective potential of naringenin and to assess the involvement of estrogenic pathway, oxidative stress, and apoptosis in relation to learning and memory deficits in an intrahippocampal $\mathrm{A} \beta$-injected rat model of $\mathrm{AD}$. 


\section{Material and methods}

\subsection{Animals}

Adult male Wistar rats (Pasteur's Institute, Tehran), weighing 240-300 g at the start of the experiment were housed three to four per cage in a temperature-controlled colony room (room temperature was $21-23^{\circ} \mathrm{C}$ ) under 12:12 light/dark cycle (lights on: 06-18). Animals were allowed to acclimate to their environment for 10 days prior to being tested and handled daily. The animals were given free access to water and kept at $80-85 \%$ of their free feeding body weight throughout the experiment. All behavioral experiments were carried out between 10 a.m. and 4 p.m. This study was conducted in accordance with the policies stipulated in the Guide for the Care and Use of Laboratory Animals (NIH) and approved by the Research Council of Iran University of Medical Sciences (Tehran, Iran).

\subsection{Experimental procedure}

Rats ( $\mathrm{n}=45)$ were randomly allocated to the following equal-sized groups: sham, naringeninpretreated sham, beta amyloid (A-beta), naringenin-pretreated $A \beta$, and naringenin-pretreated $\mathrm{A} \beta$ receiving fulvestrant as an estrogen receptor antagonist. For stereotaxic surgery, rats were anesthetized with a combination of ketamine $(80 \mathrm{mg} / \mathrm{kg}$, i.p. $)$ and xylazine $(10 \mathrm{mg} / \mathrm{kg}$, i.p. $)$ and then placed in a Stoelting stereotaxic apparatus (incisor bar $-3.3 \mathrm{~mm}$, ear bars positioned symmetrically). The scalp was cleaned with iodine solution and incised on the midline, and a burr hole was drilled through the skull and $A \beta_{1-40}$ (Sigma-Aldrich, USA) was injected at coordinates of $-3.6 \mathrm{~mm}$ posterior to bregma, $2 \mathrm{~mm}$ lateral to sagittal suture, and 2.6-2.8 $\mathrm{mm}$ below dura, according to the stereotaxic atlas (Paxinos and Watson, 1986). Naringenin (Sigma-Aldrich, USA) was dissolved in $10 \%$ Cremophor and administered orally by gavage at a dose of $100 \mathrm{mg} / \mathrm{kg}$ one hour before surgery. The dosage was chosen according to the results of our pilot study and its 
efficacy in an earlier study (Yang et al., 2014). Animals in the A $\beta$ group were bilaterally injected in the dorsal hippocampus with $4 \mu \mathrm{l}$ of a solution containing $\mathrm{A} \beta_{1-40}(2 \mathrm{nmol} / 4 \mu \mathrm{l})$. The amount of $\mathrm{A} \beta(0.5 \mathrm{nM} / \mu 1$ dissolved in $0.9 \%$ normal saline; $\mathrm{pH}=8.0)$ was chosen based on our earlier experiment, and the solution was prepared according to previous studies (Bagheri et al., 2011; Miguel-Hidalgo et al., 2002) and then immediately stored at $-70^{\circ} \mathrm{C}$ until used. Sham group received $4 \mu \mathrm{l}$ of $0.9 \%$ normal saline instead of $A \beta$ solution. The ER antagonist fulvestrant (Sigma-Aldrich, USA) was injected i.c.v. at a dose of $10 \mu \mathrm{g} / \mathrm{rat}(5 \mu \mathrm{l})$ at coordinates of $-0.8 \mathrm{~mm}$ posterior to bregma, $1.4 \mathrm{~mm}$ lateral to bregma, and $4 \mathrm{~mm}$ below dura $30 \mathrm{~min}$ before $\mathrm{A} \beta$ injection. Fulvestrant was dissolved in dimethyl sulfoxide (DMSO) and diluted to the required volume with artificial CSF (ACSF) containing the following: $120 \mathrm{mM} \mathrm{NaCl}, 3 \mathrm{mM} \mathrm{KCl}, 1.15 \mathrm{mM} \mathrm{CaCl} 2,0.8$ $\mathrm{mM} \mathrm{MgCl} 2,27 \mathrm{mM} \mathrm{NaHCO}$, and $0.33 \mathrm{mM} \mathrm{NaH2PO} 4$; $\mathrm{pH}$ adjusted to 7.2. Post-operatively, the rats were given special care until spontaneous feeding was restored. Behavioral tests were conducted after two weeks post-surgery as depicted in Fig. 1 and were evaluated blind to the treatments by the observer.

\subsection{Y maze task}

Spatial recognition memory was assessed by recording spontaneous alternation behavior in a single-session Y-maze on the $14^{\text {th }}$ day post-surgery, as described before (Baluchnejadmojarad and Roghani, 2011). The maze was made of black Plexiglas. Each arm was $40 \mathrm{~cm}$ long, $30 \mathrm{~cm}$ high and $15 \mathrm{~cm}$ wide. The arms converged in an equilateral triangular central area that was $15 \mathrm{~cm}$ at its longest axis. The procedure was as follows: each rat, naive to the maze, was placed at the end of one arm and was allowed to move freely through the maze during an 8-min session. The series of arm entries were recorded visually. Entry was considered to be complete when the base of the animal's tail was entirely within the arm. Alternation was defined as successive entries into 
the three arms on overlapping triplet sets. The maximum number of possible spontaneous alternations was determined as the total number of arms entered minus 2 , and the percentage was calculated as the ratio of actual to possible alternations $\times 100$.

\subsection{RAM task}

Spatial memory were tested using a radial arm maze (RAM) according to the paradigm described previously (Bagheri et al., 2011; Baluchnejadmojarad and Roghani, 2006). The apparatus consisted of a 50-cm-elevated (above the floor) eight-armed radial maze (RAM) made of black Plexiglas. The maze was placed in a sound-attenuated and dimly lit room. The 60-cm-long, 10cm-wide, and 15-cm-high arms extended radially from a central octagonal starting platform (35 $\mathrm{cm}$ in diameter), and there was a recessed food cup at the end of each arm. In some of the arms, the cup contained a single small food pellet as a reinforcer. A plastic cylinder $(30 \mathrm{~cm}$ in diameter, $20 \mathrm{~cm}$ high) was placed on the central platform, and a rat was placed inside this cylinder $15 \mathrm{~s}$ before the test. Following this interval, the rats were allowed to move freely and timing began. The RAM was surrounded by various extra-maze cues; their orientation relative to the maze was kept constant throughout the experiment. The maze was cleaned with diluted ethanol between trials.

Prior to acquisition (i.e., before surgery), the rats were maintained on a restricted feeding schedule designed to keep their body weight at about $85 \%$ of the free-feeding level. The rats learned to visit each arm, eat the pellet, and not re-enter the arm that had been visited during the same test. Each entry into each arm with all four paws was scored during a period of $10 \mathrm{~min}$. Behavioral observation was discontinued after $10 \mathrm{~min}$, even if the animal did not finish the task. The number of correct choices or errors was used to assess the performance of the animal in each session. An error was defined as a re-entry into an already visited arm. Rats that made at least seven correct choices in each of three consecutive sessions were used in the subsequent 
behavioral experiments. Training was performed at 24-h intervals, and rats that fulfilled the above-mentioned criteria within two weeks were included in the study. Retention trials were performed once on the $16^{\text {th }}$ day post-surgery.

\subsection{Single trial passive avoidance test}

The protocol of this test has been described before (Baluchnejadmojarad and Roghani, 2011). The apparatus $(40 \mathrm{~cm}$ long $\times 20 \mathrm{~cm}$ wide $\times 30 \mathrm{~cm}$ high $)$ consisted of an illuminated chamber connected to a dark chamber by a guillotine door. Electric shocks were delivered to the grid floor by an isolated stimulator. On the first and second days of testing, each rat was placed in the apparatus for 15 min to habituate. On the third day, an acquisition trial was performed. Rats were placed individually in the illuminated chamber. After a habituation period ( $5 \mathrm{~min}$ ), the guillotine door was lifted, and, after the rat had entered the dark chamber, the door was lowered and an inescapable scrambled single electric shock $(1 \mathrm{~mA}, 1 \mathrm{~s})$ was delivered. In this trial, the initial latency (IL) of entrance into the dark chamber was recorded, and all rats had ILs greater than $60 \mathrm{~s}$ and were included in the study. Twenty-four hours later, each rat was placed in the illuminated chamber for retention trial. The interval between placement in the illuminated chamber and entry into the dark chamber was measured as step-through latency (STL, up to a maximum of $300 \mathrm{~s}$ ). This test was conducted on days 17-20 post-surgery.

\subsection{Measurement of oxidative stress markers}

\subsubsection{Determination of hippocampal MDA concentration}

The rats were anesthetized with ketamine $(100 \mathrm{mg} / \mathrm{kg})$ and decapitated. Hippocampi were isolated and blotted dry, and then weighed and prepared as a 5\% tissue homogenate in ice-cold $0.9 \%$ saline solution. After centrifugation $\left(1000 \times \mathrm{g}, 4^{\circ} \mathrm{C}, 10 \mathrm{~min}\right)$, the supernatant was aliquoted and stored at $-70^{\circ} \mathrm{C}$ until assayed. The concentration of malondialdehyde (MDA), used as a marker of lipid peroxidation index, was calculated by measuring thiobarbituric acid reactive 
substances (TBARS) in the supernatant as described previously (Afshin-Majd et al., 2014).

Briefly, trichloroacetic acid and TBARS reagent were added to aliquots of the supernatant, which were subsequently mixed and incubated at $100^{\circ} \mathrm{C}$ for $80 \mathrm{~min}$. After cooling on ice, the samples were centrifuged at $1000 \times \mathrm{g}$ for $10 \mathrm{~min}$, and the absorbance of the supernatant was read at 532 $\mathrm{nm}$. The results of TBARS measurements were expressed as MDA equivalents, using tetraethoxypropane as standard.

\subsubsection{Measurement of hippocampal SOD activity}

Superoxide dismutase (SOD) activity was measured as previously reported (Roghani and Baluchnejadmojarad, 2009). Briefly, supernatant was incubated with xanthine and xanthine oxidase in potassium phosphate buffer $\left(\mathrm{pH} 7.8,37^{\circ} \mathrm{C}\right)$ for $40 \mathrm{~min}$, and then nitroblue tetrazolium (NBT) was added. Thereafter, blue formazan was monitored spectrophotometrically at $550 \mathrm{~nm}$. The amount of protein that inhibited NBT reduction to 50\% maximum was defined as 1 nitrite unit (NU) of SOD activity.

\subsubsection{Assay of hippocampal nitrite concentration}

Supernatant nitrite $\left(\mathrm{NO}_{2}{ }^{-}\right)$content was assayed by the Griess method, as described before (Bagheri et al., 2011). The compound NO has a short half-life and is rapidly converted to the stable end products nitrate $\left(\mathrm{NO}_{3}^{-}\right)$and $\mathrm{NO}_{2}^{-}$. In the assay used here, $\mathrm{NO}_{3}^{-}$is converted to $\mathrm{NO}_{2}^{-}$by cadmium, and this is followed by color development with Griess reagent (sulfanilamide and Nnaphthyl ethylenediamine) in acidic medium. The absorbance was determined using a spectrophotometer at $540 \mathrm{~nm}$.

\subsubsection{Protein assay}

The protein content of the supernatant was measured by the Bradford method, using bovine serum albumin (Sigma Chemical, St. Louis, MO) as the standard (Bradford, 1976).

\subsection{Determination of DNA Fragmentation (Apoptosis)}


In this experiment, the determination of histone-associated DNA fragments was performed using the Cell Death Detection ELISA kit (Roche Diagnostics, Germany) as an indicator of apoptosis according to the protocol from the company and the procedure as described before (Afshin-Majd et al., 2014). The assay is based on a quantitative sandwich-enzyme-immunoassay principle using mouse monoclonal antibodies directed against DNA and histones, respectively. This allows the specific determination of mono- and oligonucleosomes (histone-associated DNA fragments) in the fraction of tissue lysates. The amount of nucleosomes demonstrating DNA degradation was quantified by peroxidase (POD) retained in the immunocomplex. POD was determined photometrically at $405 \mathrm{~nm}$ with 2,2'-azino-bis(3-ethylbenzothiazoline-6-sulphonic acid) as a substrate by microplate reader (BioTek, USA) after $15 \mathrm{~min}$ of substrate reaction time. Values were expressed as the optical density (OD).

\subsection{Statistical Analysis}

All results were expressed as mean \pm S.E.M. The non-parametric Kruskal-Wallis test was used to analyze the behavioral data, and if a difference was found to be significant, pair-wise comparison was done using the Mann-Whitney U-test. Parametric one-way ANOVA was used to assess the biochemical results. In all calculations, a difference at $P<0.05$ was regarded as significant.

\section{Results}

\subsection{Alternation behavior in $Y$ maze task}

Fig. 2 illustrates the performance of rats in the Y-maze task, which was studied to assess spatial recognition memory. In this respect, alternation score was significantly different between the groups (Kruskal-Wallis, H (4)=18.7, $P<0.005)$. The alternation score was found to be significantly lower for $\mathrm{A} \beta$ group $(49.5 \pm 5.1 \%)$ as compared to the sham group $(81.1 \pm 5.4 \%)$ at the end of the study (Mann-Whitney, $\mathrm{U}=15.3, P<0.01$ ). Moreover, the score was significantly 
higher for naringenin-pretreated $\mathrm{A} \beta$ group $(69.4 \pm 6.7 \%)$ as compared to the animals in the $\mathrm{A} \beta$ group (Mann-Whitney, $\mathrm{U}=21.4, P<0.05$ ). The alternation score was also non-significantly higher for naringenin-pretreated $\mathrm{A} \beta$ group receiving the estrogen receptor antagonist fulvestrant $(63.7 \pm 6.3 \%)$ as compared to the $\mathrm{A} \beta$ group. In addition, the naringenin-pretreated sham group $(73.3 \pm 5.9 \%)$ had not a significant difference versus sham group regarding alternation percentage. To avoid a compounding effect of locomotor activity on the performance of the rats in memory evaluation tests, we used the total number of arms entered by rats as an index of such activity. In this respect, there were no significant differences amongst the groups (data not shown).

\subsection{Passive avoidance test}

Fig. 3 shows the performance of rats in the passive avoidance test as determined by IL and STL. For IL, no significant differences were found amongst the groups. Regarding STL, there was a significant difference between the groups (Kruskal-Wallis, $\mathrm{H}(4)=21.8, P<0.001)$. In this respect, the $\mathrm{A} \beta$ group $(23.1 \pm 5.3 \mathrm{~s} ; P<0.005)$, and the $\mathrm{A} \beta$ group pretreated with naringenin and receiving fulvestrant $(38.8 \pm 8.2 \mathrm{~s} ; P<0.01)$ showed a significant impairment of retention and recall as compared to the sham group $(99.7 \pm 13.4 \mathrm{~s})$. Moreover, naringenin-pretreated $\mathrm{A} \beta$ group showed a significant improvement of STL (65.6 $\pm 10.7 \mathrm{~s})$ as compared to A $\beta$ group (MannWhitney, $\mathrm{U}=17.3, P<0.01$ ) and this difference significantly abolished in the presence of fulvestrant $(P<0.05)$. Furthermore, STL in naringenin-pretreated sham group did not show a significant difference versus sham group.

\subsection{RAM task}

Statistical analysis showed a significant difference between the groups regarding correct choices (Kruskal-Wallis, H (4)=21.3, $P<0.005)$ and errors (Kruskal-Wallis, H $(4)=20.4, P<0.005)$. 
A $\beta$ group showed a significant deficit in spatial cognition in this task, as indicated by a lower number of correct choices (Mann-Whitney, $\mathrm{U}=15.9, P<0.01$ ) and a higher number of errors (Mann-Whitney, $\mathrm{U}=16.4, P<0.01$ ) as compared to data from sham group. Naringenin pretreatment of $\mathrm{A} \beta$ group caused a significant increase in the number of correct choices (MannWhitney, $\mathrm{U}=21.8, P<0.05$ ) and non-significantly lowered the number of errors versus $\mathrm{A} \beta$ group. Meanwhile, administration of fulvestrant to naringenin-pretreated $\mathrm{A} \beta$ group caused significant decrease (Mann-Whitney, $\mathrm{U}=16.7, P<0.01$ ) of correct choices (as observed in the A $\beta$ group) and significant increase of errors (Mann-Whitney, $\mathrm{U}=15.9, P<0.01$ ) (the same as the $\mathrm{A} \beta$ group)

versus sham group (Fig. 4). Moreover, the difference between the $A \beta+$ naringenin+fulvestrant and $A \beta+$ naringenin groups was statistically significant regarding both correct choices (MannWhitney, $\mathrm{U}=19.1, P<0.05$ ) and errors (Mann-Whitney, $\mathrm{U}=20.6, P<0.05$ ).

\subsection{Hippocampal oxidative stress and apoptosis}

A $\beta$ group exhibited a significantly higher level of MDA $(10.4 \pm 1 \mathrm{nmol} / \mathrm{mg}$ protein; $P<0.01)$ and nitrite $(9.1 \pm 0.8 \mathrm{nmol} / \mathrm{mg}$ protein; $P<0.01)$, and a significant reduction of SOD activity (3.9 \pm 0.8 unit/mg protein; $P<0.01$ ) (Fig. 5) as compared to the sham group (MDA, $6.06 \pm 0.7$ $\mathrm{nmol} / \mathrm{mg}$ protein; nitrite, $5.8 \pm 0.6 \mathrm{nmol} / \mathrm{mg}$ protein; SOD $7.2 \pm 0.6 \mathrm{unit} / \mathrm{mg}$ protein). Pretreatment of A $\beta$ group with naringenin significantly restored only the level of MDA (7.6 \pm $0.7 ; P<0.05)$ relative to $\mathrm{A} \beta$ group with no significant change of nitrite and SOD. In addition, the difference between $A \beta+$ naringenin+fulvestrant and $A \beta+$ naringenin groups was not statistically significant regarding these parameters. Measurement of chromosomal breakdown of DNA as a reliable indicator of apoptosis showed a significant increase of DNA fragmentation in $A \beta$ group $(P<0.005)$ versus sham group and naringenin pretreatment of $\mathrm{A} \beta$ group significantly reduced 
this index relative to $\mathrm{A} \beta$ group $(P<0.05)$ as shown in Fig. 6 . In addition, fulvestrant administration to $\mathrm{A} \beta+$ naringenin group abolished this significant difference.

\section{Discussion}

This study was undertaken to assess the preventive effect of naringenin on learning and memory deficits in an intrahippocampal $\mathrm{A} \beta$-injected rat model of $\mathrm{AD}$ and to determine the involvement of estrogenic receptors pathway, oxidative stress, and apoptosis in this regard. The findings of this study showed that naringenin, but not a combination of naringenin and fulverstrant as an estrogenic receptor antagonist significantly improves the performance of A $\beta$-injected rats in some behavioral tasks via lowering hippocampal MDA in addition to reducing apoptosis and part of its beneficial effect was mediated through estrogenic signaling pathway.

Earlier studies have shown that infusion of $A \beta 1-40$ into the cerebral ventricles (Nabeshima and Nitta, 1994) or its intrahippocampal injection (Bagheri et al., 2011) leads to learning and memory disturbances. In this study, we observed impaired learning and memory in rats after bilateral injection of $A \beta 1-40$ into the dorsal hippocampus, which agrees with the results of our previous

study (Bagheri et al., 2011). Previously, we have done some experiments using A $\beta 1-40$ fragment which produced a stable and consistent model of AD. This model exhibited neuronal loss in different parts of hippocampus and cognitive impairment (Bagheri et al., 2011; Bagheri et al., 2013) and for this reason we decided to use A $\beta 1-40$ fragment to induce animal model of AD in this study. Regarding the oligomeric state of $A \beta$, we have done thioflavin $T$ fluorescence assay (unpublished work) and it showed that the used $A \beta$ solution in our study contained both free and fibrillar forms of $\mathrm{A} \beta 1-40$.

Several evidence suggests that women are at a twofold risk of developing late onset AD after the age of 65 years compared to men which has been linked to endogenous estrogen deficiency, 
while estrogen replacement therapy can be effective to lower risk of AD (Alzheimer's Association, 2012). The results of previous studies suggest that estrogen replacement therapy may be associated with better mood, cognitive function and quality of life through promoting neuronal sprouting, enhancing cholinergic activity, decreasing brain and plasma levels of betaamyloid as discussed before (Almeida et al., 2006). Estrogen probably may have a protective role in cognitive decline in $\mathrm{AD}$ (Jamshed et al., 2014). Phytoestrogens have been shown to reduce AD-related pathology, potentially alleviating risk of its progression (Soni et al., 2014). Naringenin as a phytoestrogen found in foodstuffs and nutritional supplements (Helle et al., 2014) was used in our study on this foundation. There is accumulating evidence that naringenin has an estrogenic activity, in this way affecting NO production via estrogen receptors activation (via interaction with both alpha and beta types), i.e. in endothelial cells (Liu et al., 2008). As mentioned, naringenin improved the memory of $A \beta$-injected rats in our study. However, this positive effect was not observed when we used the estrogen receptor antagonist fulvestrant, which suggests that the favorable effect of naringenin that we observed may have been partly due to its estrogen-like activity.

Recent line of research emphasized the important role of brain metabolic stress and neuroinflammation as a cause of cognitive decline in AD (De Felice and Lourenco, 2015) and A $\beta$ peptide is hypothesized to stimulate microglia to initiate a typical proinflammatory phenotype in AD (Rojanathammanee et al., 2015). Naringenin could inhibit neuroinflammation via cytokine signaling 3 and exert neuroprotection with potential benefit for treatment of inflammationassociated disorders like AD (Wu et al., 2015). Naringenin also exerts protective effects against lipopolysaccharide-induced microglial activation (Wu et al., 2015). There are also reports indicating mitochondrial dysfunction with insufficient ATP synthesis and enhanced release of reactive oxygen species known generally as oxidative stress, neuroinflammation from 
dysfunction of microglia and astrocytes, abnormal ApoE4 allele protein and aberrant Tau phosphorylation play key roles in pathophysiological changes in brains of AD patients (Weinstein et al., 2013). Intrahippocampal administration of $A \beta$ is associated with increased oxidative stress and cognitive impairment (Bagheri et al., 2011). In our study, $A \beta$ administration increased lipid peroxidation as shown by a higher level of MDA and lowered SOD activity as an antioxidant system. These results support previous findings that reported significantly increased level of MDA in rodents after injection of $A \beta$ (Cetin et al., 2013). However, there are much controversy on SOD changes in AD models. In this respect, in vitro application of $\beta$-amyloid 2535 could induce neurotoxicity in rat cortical neurons by lowering activity of antioxidant defense systems like SOD (Picaud et al., 1990). Despite that, we demonstrated a beneficial effect of naringenin pretreatment in reducing the lipid peroxidation in hippocampus caused by $\mathrm{A} \beta$ injection. However, naringenin did not prevent the impairment in SOD antioxidant system, indicating that the antioxidant effect of naringenin was independent of the antioxidant status of the hippocampus. In support of our findings, a study by Heo et al showed that pretreatment of PC12 cells with naringenin could prevent the generation of the Abeta-induced reactive oxygen species and results in the decrease of Abeta toxicity in a concentration dependent manner and inhibits the Abeta-induced neurotoxic effect. In addition, the antiamnestic activity of naringenin in vivo was also shown in scopolamine model of amnesia in the passive avoidance test (Heo et al., 2004). Part of these beneficial effects of naringenin may be attributed to its ability to reduce $\mathrm{A} \beta$ level and inflammation processes in the hippocampus. In this respect, prophylactic treatment with naringenin could improve functional disturbances and mitigate the ischemic brain injury by suppressing NF-кB-mediated neuroinflammation (Raza et al., 2013).

Apoptosis, known as programmed cell death, is currently regarded as the primary form of cell death associated with AD. Apoptotic pathways are usually activated by a variety of factors 
including $A \beta$ deposition and oxidative stress and inflammatory processes and cannot be reversed once fully activated (Cheng and Li, 2014; Radi et al., 2014). Although apoptosis is considered to be one of the most promising therapeutic targets in neurodegenerative disorders like AD (Radi et al., 2014), no effective pharmacological interventions have been developed to date. In our study, naringenin pretreatment was capable to decrease hippocampal apoptosis that was consistent with its anti-apoptotic activity in the brain (Chtourou et al., 2014). However, there is some evidence showing pro-apoptotic and cytotoxic effect of naringen in cell culture (Arul and Subramanian, 2013). This controversial issue itself needs further investigation in the future.

In the current study, all behavioral assessments were done on days 14 to 20 post-surgery. The reason for that approach was that, in our preliminary studies, we had not observed any significant changes in learning and memory indices during days 7-13 after A $\beta$ injection, which indicated that injection of $A \beta$ into the hippocampus causes cumulative cell damage. In our study, the single dose of naringenin was given to the rats one hour before surgery. Regarding blood brain barrier (BBB) permeability of naringenin, Youdim et al investigated this issue in in vitro and in vivo conditions (Youdim et al., 2004). They exhibited that naringenin has high permeability across BBB through its different regions. Pharmacodynamics analysis showed that after single oral administration of naringenin at a dose of $20 \mathrm{mg} / \mathrm{kg}$, it is rapidly absorbed and can be observed in plasma 20 min after dosing. Plasma concentration reaches peak value after $3.5 \mathrm{~h}$ (Kanaze et al., 2007). It should be mentioned that the elimination half-life for naringenin is $3.93 \pm 1.01 \mathrm{~h}$ (Wan et al., 2011). Oral administration of naringenin is followed by rapid absorption as its conjugated forms. It has low bioavailability owing to extensive first-pass metabolism which is partly done by cleavage of c-ring by enzymes of intestinal bacterial enzymes leading to degradation products such as phenolic acids. In the current study, we administered naringenin about an hour before 
$A \beta 1-40$ injection, meaning that its protective effects still present after $A \beta 1-40$ exposure. In this study, we preferred to administer naringenin before lesioning the rats. The reason for this selection was that such compounds are more beneficial and more effective if given before or during the initial stages of pathological and clinical AD. In the present study, we used wellcharacterized hippocampus-dependent spatial memory tasks, Y maze and radial arm maze, in addition to passive avoidance test for assessment of learning and memory. The $\mathrm{Y}$ maze task is a specific and sensitive test of spatial recognition memory in rodents. The test relies on an innate tendency of rats to explore a novel environment. The Y maze used in this study involves no aversive stimuli and was considered suitable for evaluating memory. The specific part of the brain involved in performance of this task includes the hippocampus (Nasri et al., 2012). Since naringenin pretreatment significantly improved spatial working memory in A $\beta 1-40$-injected rats, as evidenced by increase of spontaneous alternation percentage as compared to $A \beta$-treated rats, this result suggests that naringenin at its used dose improved acquisition of the short-term memory of the $A \beta(1-40)$-treated rats in Y-maze task. In behavioral neuroscience, radial armmaze task is widely used for evaluation of the effect of drugs, stress and various other environmental factors on spatial memory (Bayat et al., 2012). A $\beta 1-40$-treated rats exposed to naringenin exhibited an improvement of working memory as compared to $A \beta 1-40$ alone-treated rats in radial arm-maze task. These findings could suggest that naringenin plays an important role in spatial memory formation.

\section{Conclusion}

Our findings suggest that naringenin pretreatment attenuates $A \beta$-induced impairment of learning and memory through mitigation of lipid peroxidation and apoptosis and its beneficial effect is somewhat mediated via estrogenic pathway. 


\section{Acknowledgements}

This work was financially supported by grants from the Cellular and Molecular Research Center at Iran University of Medical Sciences (Tehran) and Linköping University (Linköping, Sweden).

Conflict of interest: The authors declare that there are no conflicts of interest.

\section{References}

Afshin-Majd, S., Khalili, M., Roghani, M., Mehranmehr, N., Baluchnejadmojarad, T., 2014. Carnosine exerts neuroprotective effect against 6-hydroxydopamine toxicity in hemiparkinsonian rat. Mol. Neurobiol. 51, 1064-70

Almeida, O.P., Lautenschlager, N.T., Vasikaran, S., Leedman, P., Gelavis, A., Flicker, L., 2006. A 20-week randomized controlled trial of estradiol replacement therapy for women aged 70 years and older: effect on mood, cognition and quality of life. Neurobiol. Aging 27, 141-149.

Arul, D., Subramanian, P., 2013. Naringenin (citrus flavonone) induces growth inhibition, cell cycle arrest and apoptosis in human hepatocellular carcinoma cells. Pathol. Oncol. Res. 19, 763770.

Alzheimer's Association, 2012. 2012 Alzheimer's disease facts and figures. Alzheimers Dement. $8,131-168$.

Bagheri, M., Joghataei, M.T., Mohseni, S., Roghani, M., 2011. Genistein ameliorates learning and memory deficits in amyloid beta(1-40) rat model of Alzheimer's disease. Neurobiol. Learn. Mem. 95, 270-276.

Bagheri, M., Rezakhani, A., Nystrom, S., Turkina, M.V., Roghani, M., Hammarstrom, P., Mohseni, S., 2013. Amyloid beta(1-40)-induced astrogliosis and the effect of genistein treatment in rat: a three-dimensional confocal morphometric and proteomic study. PLoS One 8, e76526. 
Baluchnejadmojarad, T., Roghani, M., 2006. Effect of naringenin on intracerebroventricular streptozotocin-induced cognitive deficits in rat: a behavioral analysis. Pharmacology 78, 193197.

Baluchnejadmojarad, T., Roghani, M., 2011. Chronic epigallocatechin-3-gallate ameliorates learning and memory deficits in diabetic rats via modulation of nitric oxide and oxidative stress. Behav. Brain Res. 224, 305-310.

Bao, X.Q., Li, N., Wang, T., Kong, X.C., Tai, W.J., Sun, H., Zhang, D., 2013. FLZ alleviates the memory deficits in transgenic mouse model of Alzheimer's disease via decreasing beta-amyloid production and tau hyperphosphorylation. PloS One 8, e78033.

Bayat, M., Baluchnejadmojarad, T., Roghani, M., Goshadrou, F., Ronaghi, A., Mehdizadeh, M., 2012. Netrin-1 improves spatial memory and synaptic plasticity impairment following global ischemia in the rat. Brain Res. 1452, 185-194.

Bradford, M.M., 1976. A rapid and sensitive method for the quantitation of microgram quantities of protein utilizing the principle of protein-dye binding. Anal. Biochem. 72, 248-254.

Cetin, F., Yazihan, N., Dincer, S., Akbulut, G., 2013. The effect of intracerebroventricular injection of beta amyloid peptide (1-42) on caspase-3 activity, lipid peroxidation, nitric oxide and NOS expression in young adult and aged rat brain. Turk. Neurosurg. 23, 144-150.

Cheng, X.L., Li, M.K., 2014. Effect of topiramate on apoptosis-related protein expression of hippocampus in model rats with Alzheimers disease. Eur. Rev. Med. Pharmacol. Sci. 18, 761768.

Chtourou, Y., Fetoui, H., Gdoura, R., 2014. Protective effects of naringenin on iron-overloadinduced cerebral cortex neurotoxicity correlated with oxidative stress. Biol. Trace Elem. Res. $158,376-383$. 
De Felice, F.G., Lourenco, M.V., 2015. Brain metabolic stress and neuroinflammation at the basis of cognitive impairment in Alzheimer's disease. Front. Aging Neurosci. 7, 94.

Esmaeili, M.A., Alilou, M., 2014. Naringenin attenuates CCl4 -induced hepatic inflammation by the activation of an Nrf2-mediated pathway in rats. Clin. Exp. Pharmacol. Physiol. 41, 416-422. Grothe, M.J., Schuster, C., Bauer, F., Heinsen, H., Prudlo, J., Teipel, S.J., 2014. Atrophy of the cholinergic basal forebrain in dementia with Lewy bodies and Alzheimer's disease dementia. J. Neurol. 261, 1939-1948.

Helle, J., Kraker, K., Bader, M.I., Keiler, A.M., Zierau, O., Vollmer, G., Welsh, J., Kretzschmar, G., 2014. Assessment of the proliferative capacity of the flavanones 8-prenylnaringenin, 6-(1.1dimethylallyl)naringenin and naringenin in MCF-7 cells and the rat mammary gland. Mol. Cell Endocrinol. 392, 125-135.

Heo, H.J., Kim, D.O., Shin, S.C., Kim, M.J., Kim, B.G., Shin, D.H., 2004. Effect of antioxidant flavanone, naringenin, from Citrus junoson neuroprotection. J. Agric. Food Chem. 52, 15201525.

Jamshed, N., Ozair, F.F., Aggarwal, P., Ekka, M., 2014. Alzheimer disease in post-menopausal women: Intervene in the critical window period. J. Midlife Health 5, 38-40.

Kanaze, F.I., Bounartzi, M.I., Georgarakis, M., Niopas, I., 2007. Pharmacokinetics of the citrus flavanone aglycones hesperetin and naringenin after single oral administration in human subjects. Eur. J. Clin. Nutr. 61, 472-477.

Khan, M.B., Khan, M.M., Khan, A., Ahmed, M.E., Ishrat, T., Tabassum, R., Vaibhav, K., Ahmad, A., Islam, F., 2012. Naringenin ameliorates Alzheimer's disease (AD)-type neurodegeneration with cognitive impairment (AD-TNDCI) caused by the intracerebroventricular-streptozotocin in rat model. Neurochem. Int. 61, 1081-1093. 
Liu, L., Xu, D.M., Cheng, Y.Y., 2008. Distinct effects of naringenin and hesperetin on nitric oxide production from endothelial cells. J. Agric. Food Chem. 56, 824-829.

Lou, H., Jing, X., Wei, X., Shi, H., Ren, D., Zhang, X., 2014. Naringenin protects against 6OHDA-induced neurotoxicity via activation of the Nrf2/ARE signaling pathway. Neuropharmacology 79, 380-388.

Miguel-Hidalgo, J.J., Alvarez, X.A., Cacabelos, R., Quack, G., 2002. Neuroprotection by memantine against neurodegeneration induced by beta-amyloid(1-40). Brain Res. 958, 210-221. Mimura, M., 2008. Memory impairment and awareness of memory deficits in early-stage Alzheimer's disease. Tohoku J. Exp. Med. 215, 133-140.

Nabeshima, T., Nitta, A., 1994. Memory impairment and neuronal dysfunction induced by betaamyloid protein in rats. Tohoku J. Exp. Med. 174, 241-249.

Nasri, S., Roghani, M., Baluchnejadmojarad, T., Balvardi, M., Rabani, T., 2012. Chronic cyanidin-3-glucoside administration improves short-term spatial recognition memory but not passive avoidance learning and memory in streptozotocin-diabetic rats. Phytother. Res. 26, 12051210.

Obulesu, M., Jhansilakshmi, M., 2014. Neuroinflammation in Alzheimer's disease: an understanding of physiology and pathology. Int. J. Neurosci. 124, 227-235.

Paxinos, G., Watson, C., 1986. The rat brain in stereotaxic coordinates.(2nd ed.). Academic Press, New York.

Picaud, A., Walter, P., de Preville, G., Nicolas, P., 1990. Fatal toxic hepatitis in pregnancy. A discussion of the role of methyldopa]. J. Gynecol. Obstet. Biol. Reprod. 19, 192-196. Radi, E., Formichi, P., Battisti, C., Federico, A., 2014. Apoptosis and Oxidative Stress in Neurodegenerative Diseases. J. Alzheimers Dis. 42, S125-152. 
Rahigude, A., Bhutada, P., Kaulaskar, S., Aswar, M., Otari, K., 2012. Participation of antioxidant and cholinergic system in protective effect of naringenin against type- 2 diabetes-induced memory dysfunction in rats. Neuroscience 226, 62-72.

Raza, S.S., Khan, M.M., Ahmad, A., Ashafaq, M., Islam, F., Wagner, A.P., Safhi, M.M., Islam, F., 2013. Neuroprotective effect of naringenin is mediated through suppression of NF-kappaB signaling pathway in experimental stroke. Neuroscience 230, 157-171.

Roghani, M., Baluchnejadmojarad, T., 2009. Chronic epigallocatechin-gallate improves aortic reactivity of diabetic rats: underlying mechanisms. Vascul. Pharmacol. 51, 84-89.

Rojanathammanee, L., Floden, A.M., Manocha, G.D., Combs, C.K., 2015. Attenuation of microglial activation in a mouse model of Alzheimer's disease via NFAT inhibition. J. Neuroinflammation 12, 42.

Soni, M., Rahardjo, T.B., Soekardi, R., Sulistyowati, Y., Lestariningsih, Yesufu-Udechuku, A., Irsan, A., Hogervorst, E., 2014. Phytoestrogens and cognitive function: a review. Maturitas 77, 209-220.

Subash, S., Essa, M.M., Al-Asmi, A., Al-Adawi, S., Vaishnav, R., 2014. Chronic dietary supplementation of $4 \%$ figs on the modification of oxidative stress in Alzheimer's disease transgenic mouse model. Biomed. Res. Int. 2014, 546357.

Wan, L., Sun, X., Wang, X., Li, Y., Yu, Q., Guo, C., 2011. A stereospecific HPLC method and its application in determination of pharmacokinetics profile of two enantiomers of naringenin in rats. J. Chromatogr. Sci. 49, 316-320.

Weinstein, J.D., Gonzalez, E.R., Egleton, R.D., Hunt, D.A., 2013. A paradigm shift for evaluating pharmacotherapy for Alzheimer's disease: the 10-patient screening protocol. Consult. Pharm. 28, 443-454. 
Wu, L.H., Lin, C., Lin, H.Y., Liu, Y.S., Wu, C.Y., Tsai, C.F., Chang, P.C., Yeh, W.L., Lu, D.Y., 2015. Naringenin suppresses neuroinflammatory responses through inducing suppressor of cytokine signaling 3 expression. Mol. Neurobiol., in press.

Yang, W., Ma, J., Liu, Z., Lu, Y., Hu, B., Yu, H., 2014. Effect of naringenin on brain insulin signaling and cognitive functions in ICV-STZ induced dementia model of rats. Neurol. Sci. 35, $741-751$

Youdim, K.A., Qaiser, M.Z., Begley, D.J., Rice-Evans, C.A., Abbott, N.J., 2004. Flavonoid permeability across an in situ model of the blood-brain barrier. Free Radic. Biol. Med. 36, 592604.

Zbarsky, V., Datla, K.P., Parkar, S., Rai, D.K., Aruoma, O.I., Dexter, D.T., 2005.

Neuroprotective properties of the natural phenolic antioxidants curcumin and naringenin but not quercetin and fisetin in a 6-OHDA model of Parkinson's disease. Free Radic. Res. 39, 1119-1125. Zhu, M., Xiao, S., Li, G., Li, X., Tang, M., Yang, S., Xu, X., Feng, L., Liu, K., Hu, L., 2013. Effectiveness and safety of generic memantine hydrochloride manufactured in China in the treatment of moderate to severe Alzheimer's disease: a multicenter, double-blind randomized controlled trial. Shanghai Arch. Psychiatry 25, 244-253. 


\section{Figure Legends}

Fig. 1. Experimental scheme for treatments and behavioral tests. RAM stand for radial arm maze.

Fig. 2. Alternation behavior in $Y$-maze task $(n=8-9$ for each group). Values are means \pm S.E.M. $* P<0.01$ (vs. sham); \# $P<0.05$ (vs. A-beta).

Fig. 3. Initial (IL) and step-through (STL) latencies in single-trial passive avoidance test $(n=8-9$ for each group). Values are means \pm S.E.M.

$* P<0.01, * * P<0.005$ (vs. sham); \# $P<0.01$ (vs. A-beta)

Fig. 4. Number of correct choices or the number of errors in RAM task ( $n=7-8$ for each group). Values are means \pm S.E.M. $* P<0.05, * * P<0.01$ (vs. sham); \# $P<0.01$ (vs. A-beta).

Fig. 5. Malondialdehyde (MDA) and nitrite content and SOD activity in hippocampal homogenate ( $n=6$ for each group). Values are means \pm S.E.M.

$* P<0.05, * * P<0.01$ (vs. sham); \# $P<0.05$ (vs. A-beta)

Fig. 6. Optical density for DNA fragmentation ( $n=6$ for each group). Values are means \pm S.E.M. $* P<0.01, * * P<0.005$ (vs. sham); \# $P<0.05$ (vs. A-beta) 


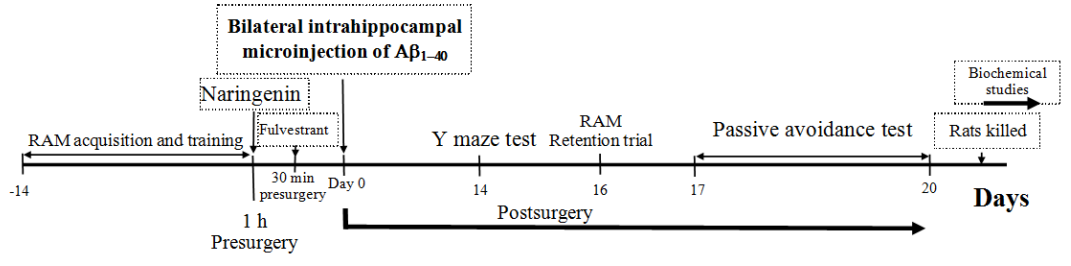

Fig. 1: 


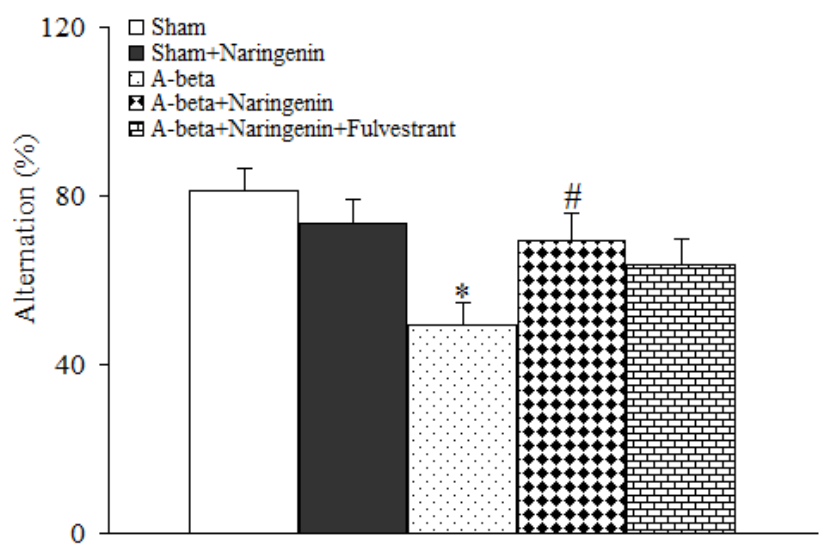

Fig. 2: 


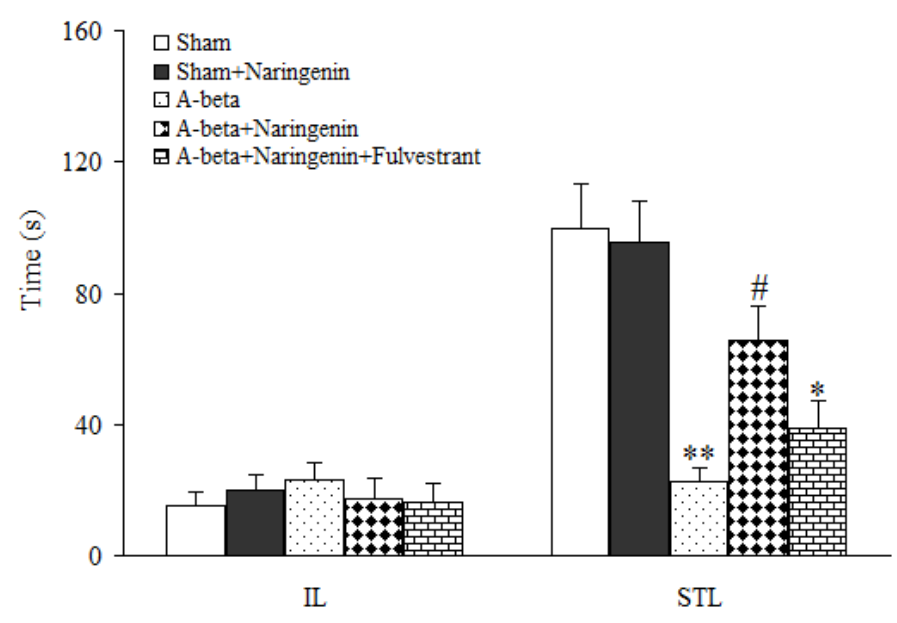

Fig. 3: 


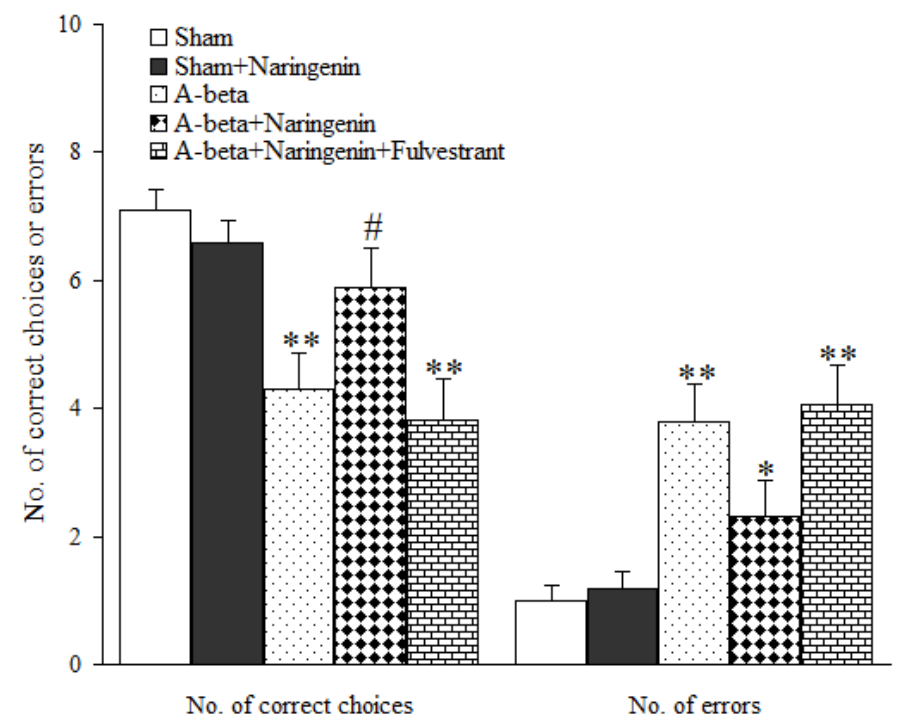

Fig. 4: 

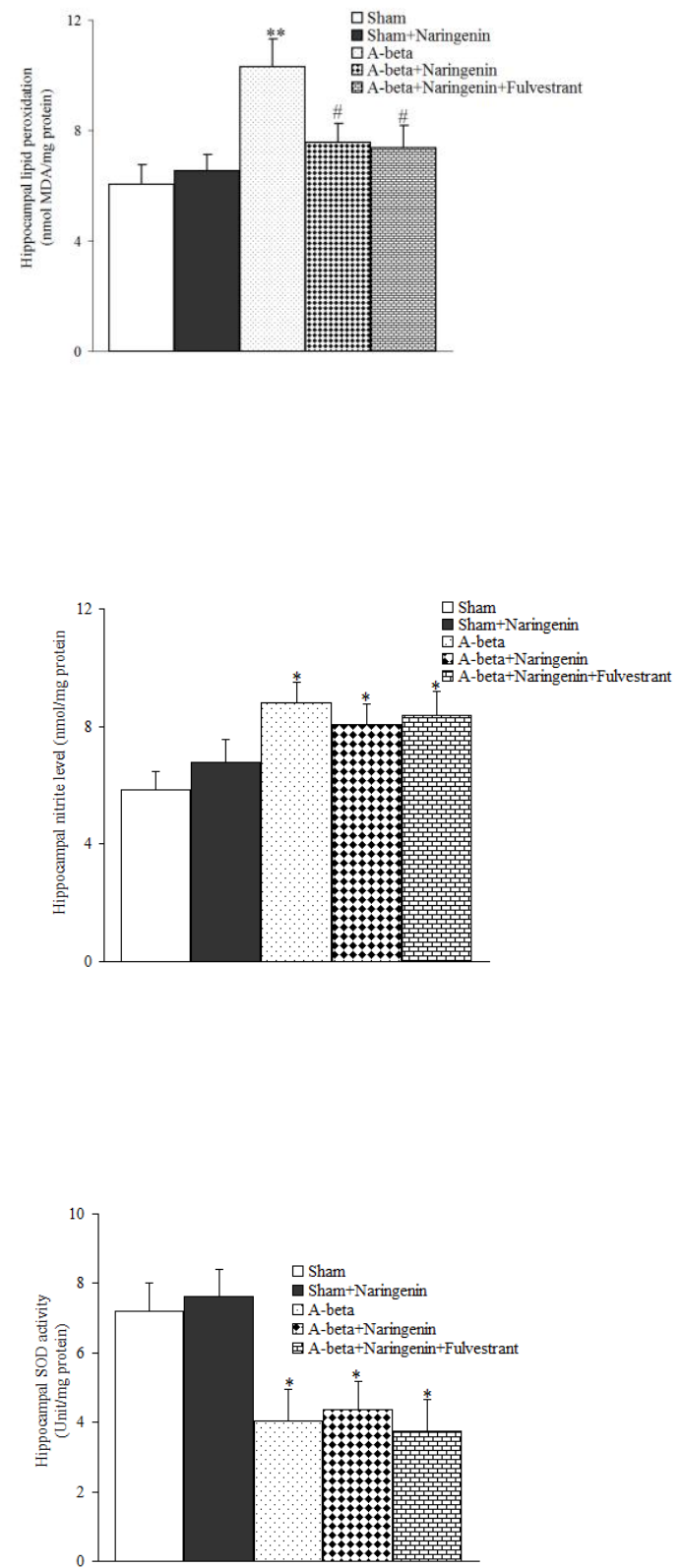

Fig. 5: 


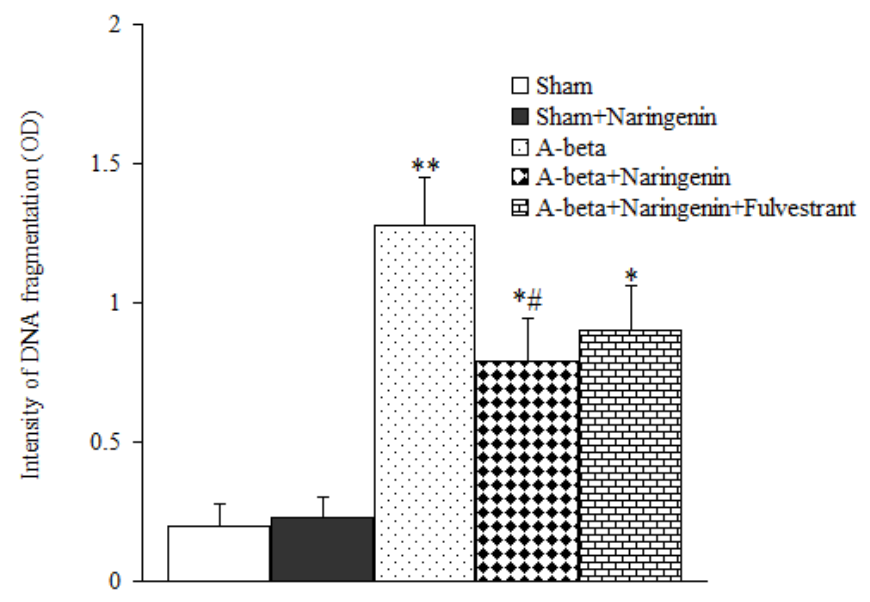

Fig. 6: 